\title{
Infective endocarditis caused by Enterobacteriaceae: phenotypic and molecular characterization of Escherichia coli and Klebsiella pneumoniae in Rio de Janeiro, Brazil
}

\author{
Nathália L. Andrade ${ }^{1}$ - Ana Carolina da Cruz Campos ${ }^{2} \cdot$ Andrea Maria Cabral $^{3}$. Paula Hesselberg Damasco ${ }^{4}$. \\ Jerome Lo-Ten-Foe ${ }^{2}$ Ana Cláudia P. Rosa ${ }^{1}$ - Paulo V. Damasco ${ }^{3,5}$
}

Received: 19 June 2020 / Accepted: 12 May 2021 / Published online: 22 September 2021

(C) The Author(s) 2021

\begin{abstract}
The etiological agent for infective endocarditis (IE), a life-threatening disease, is usually gram-positive bacteria. However, gram-negative bacteria can rarely cause IE and $4 \%$ of cases are associated with morbidity and mortality. This study aimed to characterize Escherichia coli and Klebsiella pneumoniae isolates from the blood of patients with IE. The characteristics of blood isolates were compared with those of urinary isolates from patients with urinary tract infections (UTIs). The results of this study revealed that $K$. pneumoniae isolates from patients with IE were phylogenetically related to those from patients with UTI. Additionally, the resistance phenotype, resistance gene, virulence gene, and plasmid profiles were similar between the blood and urinary isolates. The isolates belonging to the sequence types (STs) 76, 36, 101 (K. pneumoniae), and 69 (E. coli) are reported to be associated with drug resistance. The Enterobacteriaceae isolates from patients with IE did not produce extended-spectrum $\beta$-lactamase or carbapenemase. Additionally, this study investigated the virulence phenotype, biofilm formation ability, and the ability to adhere to the epithelial cells in vitro of the isolates. The isolates from patients with IE exhibited weaker biofilm formation ability than the urinary isolates. All isolates from patients with IE could adhere to the renal epithelial cells. However, three isolates from patients with UTIs could not adhere to the epithelial cells. The closely related $K$. pneumoniae isolates $(648, \mathrm{KP} 1, \mathrm{KP} 2, \mathrm{KP} 3$, and KP4) could not form biofilms or adhere to the epithelial cells. In summary, the molecular analysis revealed that the genetic characteristics of IE-causing K. pneumoniae and E. coli were similar to those of UTI-causing isolates. These isolates belonged to the STs that are considered treatable. Genetically similar isolates did not exhibit the same virulence phenotype. Thus, these non-hypervirulent clones must be monitored as they can cause complex infections in susceptible hosts.
\end{abstract}

Keywords Endocarditis $\cdot$ Enterobacteriaceae $\cdot$ Biofilm $\cdot$ Adhesion $\cdot$ Next-generation sequencing $\cdot$ Brazil

\section{Abbreviations \\ IE Infective endocarditis \\ UTIs Urinary tract infections \\ CAIE Community-associated IE}

HAIE Healthcare-associated IE

MDR Multidrug-resistant
Responsible Editor: Tânia A. Tardelli Gomes

Ana Carolina da Cruz Campos

anabio86@gmail.com; a.c.da.cruz.campos@rug.nl

Ana Cláudia P. Rosa

anarosa2004@gmail.com

1 Department of Microbiology, Immunology and Parasitology, Biomedical Center, Rio de Janeiro State University, Blv 28 de Setembro, 87, 3th floor, Vila Isabel, Rio de Janeiro, Brazil

2 Department of Medical Microbiology and Infection Prevention, University of Groningen, University Medical
Center Groningen, EB80 Hanzeplein 1, 9713, GZ, Groningen, The Netherlands

3 Pedro Ernesto University Hospital, Rio de Janeiro State University, Vila Isabel, Rio de Janeiro, Brazil

4 Antônio Pedro University Hospital, Federal Fluminense University, Niterói, Brazil

5 Department of Infectious and Parasitic Diseases, Graffrée e Guinle University Hospital, Federal University of the State of Rio de Janeiro, Rio de Janeiro, Brazil 


\section{Introduction}

Infective endocarditis (IE), a systemic life-threatening infection, requires a multidisciplinary therapeutic strategy $[1,2]$. The incidence of IE, which is a rare pathological condition, has increased in developed countries but there are limited studies on IE in developing countries [2, 3]. Endocarditis is the fourth most common life-threatening infectious syndrome after urosepsis, pneumonia, and intraabdominal sepsis in the medical facilities of developed countries [3].

Gram-negative bacteria that cause IE are traditionally classified into the following two main categories: HACEK group (Haemophilus spp., Aggregatibacter spp., Cardiobacterium hominis, Eikenella corrodens, and Kingella kingae) and non-HACEK group (mostly Pseudomonas spp. and members of the Enterobacteriaceae family) [4, 5]. Escherichia coli and Klebsiella pneumoniae are the etiological agents for community-associated and nosocomial infections, especially bloodstream infections and urinary tract infections (UTIs) [6-8]. Recently, there is an increased incidence of invasive infections caused by $E$. coli and $K$. pneumoniae [7-9]. The treatment of $E$. coli and $K$. pneumoniae infections is challenging owing to the development of multidrug resistance in the pathogens [10,11]. In particular, drug resistance is a major challenge to treat virulent gram-negative bacterial infections [5, 7]. Several studies have analyzed the pathophysiology of the virulence and the mechanisms underlying host cell adhesion of grampositive microorganisms in IE. However, the pathological mechanisms of IE caused by the members of the family Enterobacteriaceae have not been completely elucidated [2-5].

This study aimed to comparatively analyze the molecular characteristics of E. coli and K. pneumoniae isolated from the blood of patients with IE in Rio de Janeiro, Brazil, with those of other pathogens isolated from the urine of hospitalized patients with UTIs. Additionally, the virulence mechanisms of adherence to host cells were investigated using two different cell lines. Furthermore, the biofilm formation ability of the blood and urinary isolates was examined. The findings of this study will provide novel insights into IE caused by $E$. coli and $K$. pneumoniae, especially multidrug-resistant (MDR) pathogens.

\section{Material and methods}

\section{Bacterial strains}

In this study, five isolates from patients with IE were investigated. The E. coli isolates were obtained from the blood samples of an elderly female patient with community-associated IE (CAIE) at the Pedro Ernesto University Hospital (HUPE/UERJ). The K. pneumoniae isolates (KP1, KP2, KP3, and KP4) were obtained from the blood samples of an elderly male patient with renal failure and classical healthcare-associated endocarditis (HAIE) at the Hospital of the Public Network, Ordem Terceira do Carmo Hospital, Rio de Janeiro, Brazil. Other pathogenic $K$. pneumoniae strains $(1076,648$, 2801, and 5459) were obtained from the urine of hospitalized patients with UTI at the HUPE/UERJ. The EAEC042 and UPECI64 strains were used as positive controls for biofilm formation and adherence assays, respectively. The DH5 $\alpha$ strain was used as the negative control in these assays [12]. The sequences of the $E$. coli strains UMN026 (National Center for Biotechnological Information (NCBI) reference sequence: NC_011751.1), JJ1886 (NCBI reference sequence: CP006784.1), UTI89 (NCBI reference sequence: NC_007946.1), 536 (NCBI reference sequence: NC_008253.1), and MG1655 (NCBI reference sequence: NZ_CP032667.1) were downloaded from the NCBI database and used as a control for molecular analysis (Table 1).

\section{Strain isolation, identification, culture conditions, and susceptibility test}

The strains were plated onto the following selective media: eosin methylene-blue agar (Bevton \& Dickson, NJ, USA) and MacConkey agar (Becton \& Dickson, NJ, USA). Biochemical and matrix-assisted laser desorption/ ionization time-of-flight mass spectrometry (Bruker, Germany) analyses were performed to identify the strains. The strains were stored in GC medium with $20 \%$ glycerol at $-20^{\circ} \mathrm{C}$ and $-70{ }^{\circ} \mathrm{C}$. The isolates were cultured in blood agar medium for $18 \mathrm{~h}$ at $37{ }^{\circ} \mathrm{C}$. The drug susceptibility assays were performed using VITEK-2 (bioMérieux, Marcy l'Etoile, France), following the European Committee on Antimicrobial Susceptibility Testing guidelines and the results were confirmed using the E-test (bioMérieux) assays. The antimicrobial resistance classification of isolates was defined according to the standardized international terminology established by the European Centre for Disease Prevention and Control and the Centers for Disease Control and Prevention as follows: MDR, non-susceptibility to at least one agent in three or more antimicrobial categories; extensively drug-resistant (XDR), non-susceptibility to at least one agent in all but two or fewer antimicrobial categories; pan-drug-resistant, non-susceptibility to all agents in all antimicrobial categories [13]. 
Table 1 Bacterial isolates, resistance phenotype, resistance, and virulence genotype and adhesion results

\begin{tabular}{|c|c|c|c|c|c|c|c|c|}
\hline Isolates & Species & Source & Hospital & $\begin{array}{l}\text { Resistance phe- } \\
\text { notype }\end{array}$ & $\begin{array}{l}\text { Resistance geno- } \\
\text { type }\end{array}$ & $\begin{array}{l}\text { Virulence geno- } \\
\text { type }\end{array}$ & Adherence Vero & Adherence HEp2 \\
\hline DO7785 & E. coli & Blood & HUPE & $\begin{array}{l}\text { Ampicillin, } \\
\text { trimethoprim, } \\
\text { trimethoprim } \\
\text { sulfamethoxazole }\end{array}$ & $\begin{array}{l}\text { strA, strB, aadA5, } \\
\quad \text { bla }_{T E M-1 B}, \text { sull }, \\
\text { sul2, and } d f r A 17\end{array}$ & $\begin{array}{l}\text { Iuat, alls, fimH, } \\
\text { OmpA, irp2 }\end{array}$ & Adhere & Adhere \\
\hline KP1 & K. pneumoniae & Blood & OTC & $\begin{array}{l}\text { Ampicillin, nitro- } \\
\text { furantoin, and } \\
\text { fosfomycin }\end{array}$ & $\begin{array}{l}b^{b l a_{S H V-1}, o q x B}, \\
o q x A, \text { and fosA }\end{array}$ & $\begin{array}{l}\text { mrkD, ureA, uge, } \\
\text { pgaC, wzi, fimC, } \\
\text { OmpA, wabG, } \\
\text { hgpA }\end{array}$ & Adhere & Adhere \\
\hline KP2 & K. pneumoniae & Blood & OTC & $\begin{array}{l}\text { Ampicillin, nitro- } \\
\text { furantoin, and } \\
\text { fosfomycin }\end{array}$ & $\begin{array}{l}b l a_{S H V-1}, o q x B, \\
o q x A, \text { and fosA }\end{array}$ & $\begin{array}{l}\text { mrkD, ureA, uge, } \\
\text { pgaC, wzi, fimC, } \\
\text { OmpA, wabG, } \\
\text { hgpA }\end{array}$ & Adhere & Adhere \\
\hline KP3 & K. pneumoniae & Blood & OTC & $\begin{array}{l}\text { Ampicillin, nitro- } \\
\text { furantoin, and } \\
\text { fosfomycin }\end{array}$ & $\begin{array}{l}b^{b l a_{S H V-1}, o q x B}, \\
o q x A, \text { and fosA }\end{array}$ & $\begin{array}{l}\text { mrkD, ureA, uge, } \\
\text { pgaC, wzi, fimC, } \\
\text { OmpA, wabG, } \\
\text { hgpA }\end{array}$ & Adhere & Adhere \\
\hline KP4 & K. pneumoniae & Blood & OTC & $\begin{array}{l}\text { Ampicillin, nitro- } \\
\text { furantoin, and } \\
\text { fosfomycin }\end{array}$ & $\begin{array}{l}b^{b l a_{S H V-1}, o q x B}, \\
o q x A, \text { and fosA }\end{array}$ & $\begin{array}{l}\text { mrkD, ureA, uge, } \\
\text { pgaC, wzi, fimC, } \\
\text { OmpA, wabG, } \\
\text { hgpA }\end{array}$ & Adhere & Adhere \\
\hline 2801 & K. pneumoniae & Urine & HUPE & $\begin{array}{l}\text { Cefuroxime, } \\
\text { Cefotaxime, } \\
\text { ceftazidime, } \\
\text { gentamicin, } \\
\text { tobramycin, } \\
\text { ciprofloxacin, } \\
\text { nitrofurantoin, } \\
\text { fosfomycin, and } \\
\text { trimethoprim }\end{array}$ & $\begin{array}{l}\text { bla }_{\text {TEM-1B }}, \\
\quad \text { bla }_{\text {CTXM-15 }}, \\
\text { bla }_{\text {OXA-1 }}, \text { oqxA, } \\
\text { oqxB, fosA, } \\
\text { aac }(3) \text { lld }, \text { aac }\left(6^{\prime}\right) \\
\text { lb-cr, catB4, } \\
\text { tet }(D), \text { dfrA14, } \\
\text { and } a c r R\end{array}$ & $\begin{array}{c}y b t S, \text { mrkD, ureA, } \\
\text { uge, pgaC, wzi, } \\
\text { OmpA, wabG, } \\
\text { hgpA, irp2 }\end{array}$ & Adhere & Adhere \\
\hline 5459 & K. pneumoniae & Urine & HUPE & $\begin{array}{l}\text { Ampicillin and } \\
\text { nitrofurantoin }\end{array}$ & $\begin{array}{r}b^{\prime} a_{O X A-10}, o q x B, \\
o q x A, \text { and } \text { fos } A\end{array}$ & $\begin{array}{l}\text { mrkD, ureA, uge, } \\
\text { pgaC, wzi, fimC, } \\
\text { OmpA, wabG, } \\
\text { hgpA }\end{array}$ & Adhere & Adhere \\
\hline 648 & K. pneumoniae & Urine & DASA & $\begin{array}{l}\text { Cefuroxime, } \\
\text { cefotaxime, } \\
\text { ceftazidime, } \\
\text { gentamicin, } \\
\text { tobramycin, } \\
\text { ciprofloxacin, } \\
\text { nitrofurantoin, } \\
\text { fosfomycin, and } \\
\text { trimethoprim }\end{array}$ & $\begin{array}{l}\text { bla }_{S H V-1}, \\
\text { bla }_{C T X M-164}, \\
\text { oqxB, oqxA, } \\
\text { fosA, and } \text { acrR }\end{array}$ & $\begin{array}{l}\text { mrkD, ureA, uge, } \\
\text { pgaC, wzi, fimC, } \\
\text { OmpA, wabG, } \\
\text { hgpA }\end{array}$ & Non-adhere & Non-adhere \\
\hline 1076 & K. pneumoniae & Urine & HUPE & Amoxicillin & $\begin{array}{l}b l a_{S H V-11}, o q x B \\
o q x A, \text { and } f o s A 5\end{array}$ & $\begin{array}{l}\text { mrkD, ureA, uge, } \\
\text { pgaC, wzi, fimC, } \\
\text { OmpA, wabG, } \\
\text { hgpA }\end{array}$ & Adhere & Adhere \\
\hline
\end{tabular}

HUPE, Pedro Ernesto University Hospital; OTC, Ordem Primeira do Carmo Hospital; DASA, Diagnóstico da América

\section{DNA extraction, whole-genome sequencing, assembly, and annotation}

Total bacterial DNA was extracted from each isolate using the Ultraclean ${ }^{\circledR}$ microbial DNA isolation kit (MO BIO Laboratories, Carlsbad, CA, USA), following the manufacturer's instructions. The
DNA library was prepared using the Illumina Nextera XT kit. The sequences were assembled using CLC Genomics Workbench v10.0.1 (CLC bio A/S, Aarhus, Denmark) with default settings and optimal word size. The assembled sequences were annotated using RAST server version 2.0 [14] (see Supplementary Data $\mathrm{S} 1)$. 


\section{Sequencing data analysis}

The assembled genomes were uploaded in the FASTA format to the Center for Genomic Epidemiology (CGE) multilocus sequence typing (MLST) finder website (version 1.7) to identify the sequence types (STs) of the isolates [15]. The presence of antibiotic-resistant genes was determined by uploading the assembled genomes in FASTA format to ResFinder 2.1 [16] from the CGE server. The virulence genes were identified using the Basic Local Alignment Search Tool of the NCBI or European Nucleotide Archive database with the CLC Genomics Workbench v10.0.1 (CLC bio A/S, Aarhus, Denmark) tool (see the complete list of virulence genes in Supplementary Data S1 and S2). The serotypes of K. pneumoniae and E. coli isolates were predicted using the Kaptive web tool [17] and SerotypeFinder tool, respectively [18]. To determine the phylogenetic characteristics, the sequences were uploaded into SeqSphere v.4.1.9 (Ridom, Munster, Germany) and genotyped using a gene-bygene typing approach with a 2358-gene core-genome MLST (cgMLST) scheme. The plasmid replicon types were identified by uploading the genome sequences of the isolate to the PlasmidFinder v.2.0.1 [19] webtool. The plasmids in the isolates were reconstructed using the MOB-suite tool [20].

\section{Cell lines and cell culture conditions}

The Vero cell line (American Type Culture Collection (ATCC) CCL-81; derived from African green monkey kidney) was used to mimic the normal human renal epithelial cells. The HEp-2 cell line (ATCC CCL 23; derived from human laryngeal epithelial cells) was used as a positive control for the adherence assay [21]. The Vero and HEp-2 cells were cultured in minimum essential medium (MEM, GibcoBRL) supplemented with 5\% v/v fetal calf serum (GibcoBRL), D-mannose, $50 \mu \mathrm{g} / \mathrm{mL}$ gentamicin, and $2.5 \mu \mathrm{g} / \mathrm{mL}$ amphotericin B. The cells were cultured on 13-mm diameter glass coverslips placed in 24-well tissue culture plates (Costar) to obtain the sub-confluent cell monolayer. Next, the monolayer was washed twice with Dulbecco's phosphatebuffered saline (PBS-D; pH 7.2) and incubated with $1 \mathrm{~mL}$ of fresh MEM without antibiotics.

\section{Adherence assays}

The cell monolayers were incubated with $35 \mu \mathrm{L}$ of bacterial culture cultured overnight in Luria-Bertani (LB) medium for 3 or $6 \mathrm{~h}$ at $37^{\circ} \mathrm{C}$ and $5 \% \mathrm{CO}_{2}$. In the 6-h assay, the cells were washed with PBS-D and fresh medium was added after $3 \mathrm{~h}$. The cells were washed twice with PBS-D to remove the non-adherent bacteria, fixed with methanol, and stained with $5 \%$ Giemsa stain for $30 \mathrm{~min}$. The coverslips were removed from the wells and the cells were washed with water, dried, mounted on glass slides, and examined using oil immersion microscopy [22].

\section{Quantitative biofilm formation assay}

The ability of E. coli and $K$. pneumoniae strains to form biofilms on polystyrene surfaces was examined according to the methodology of Sheikh et al. (2001) [12] with modifications. The bacterial suspension $(5 \mu \mathrm{L})$ cultured overnight in $\mathrm{LB}$ medium at $37^{\circ} \mathrm{C}$ with shaking was incubated with $200 \mu \mathrm{L}$ of Dulbecco's minimal essential medium (D-MEM, Gibco-BRL) in 96-well microtiter plates at $37^{\circ} \mathrm{C}$ overnight. The culture was rinsed twice with $200 \mu \mathrm{L}$ PBS to remove the planktonic cells. The biofilm was stained with crystal violet and the stain was solubilized with $200 \mu \mathrm{L}$ of $95 \%$ ethanol for $2 \mathrm{~min}$ at $37{ }^{\circ} \mathrm{C}$ room temperature. Next, $150 \mu \mathrm{L}$ of the mixture was transferred to a new microtiter plate and the absorbance at $570 \mathrm{~nm}$ was measured using an enzyme-linked immunosorbent assay plate reader. The assay was performed in triplicate and three independent experiments were performed [23]. The average optical density (OD) was calculated for all strains, including the negative control (DH5 $\alpha$ strain), according to the criteria reported by Stepanovic et al. [23]. The cut-off value was three standard deviations (SDs) from the mean OD of the negative control (ODc average $=\mathrm{OD}$ of negative control $(3 \times \mathrm{SD}$ of negative control)). To interpret the results, the strains were divided into the following categories: no biofilm producer $(0)$, weak biofilm producer $(+$ or 1$)$, moderate biofilm producer $(++$ or $2)$, and strong biofilm producer $(+++$ or 3$)$. This classification was based on the following criteria: $\mathrm{OD} \leq \mathrm{ODc}$, no biofilm producer; ODc $<\mathrm{OD} \leq 2 \times \mathrm{ODc}$, weak biofilm producer; $2 \times \mathrm{ODc}<\mathrm{OD} \leq 4 \times \mathrm{ODc}$, moderate biofilm producer; $4 \times \mathrm{ODc}<\mathrm{OD}$, strong biofilm producer. All data were analyzed using GraphPad Prism. The data were analyzed using one-way analysis of variance. The differences were considered significant at $p<0.0001$.

\section{Nucleotide sequence accession number}

The sequencing data of isolates were deposited in the NCBI public database (project number: PRJNA657729) (see Supplementary Data S3 for individual accession numbers).

\section{Results}

\section{Resistance phenotype}

The origin and species of the strains used in this study are listed in Table 1. The E. coli isolate DO7785 was resistant to trimethoprim, trimethoprim/sulfamethoxazole, and ampicillin. Six isolates were classified as MDR in this study (the 
exceptions were the isolates 5459 and 1076). KP1, KP2, $\mathrm{KP} 3$, and KP4 were resistant to ampicillin, nitrofurantoin, and fosfomycin. The isolate 1076 was resistant to amoxicillin, whereas the isolates 2801 and 648 were resistant to piperacillin/tazobactam, cefuroxime, cefotaxime, ceftazidime, gentamicin, tobramycin, ciprofloxacin, nitrofurantoin, fosfomycin, trimethoprim, and ciprofloxacin. The isolate 5459 was resistant to ampicillin and nitrofurantoin (Table 1).

\section{Detection of antibiotic-resistant genes}

The E. coli isolate DO7785 harbored the following resistance genes: strA, aadA5, strB, bla $a_{T E M-1 B}$, sull, sul2, and dfrA17. The KP1, KP2, KP3, and KP4 isolates harbored the following resistance genes: $b_{S H V-1}, o q x B, o q x A$, and fosA. The isolate 1076 harbored the following resistance genes: $b l a_{T E M-1 B}, o q x B, o q x A$, and fosA5. The isolate 648 harbored the following resistance genes: $b l a_{S H V-1}, b l a_{S H V-164}, o q x B$, $o q x A$, fosA, and acrR. The isolate 5459 harbored the following resistance genes: bla $a_{\text {OXA-10 }}$, oqXB, oqxA, and fosA. The isolate 2801 harbored the following resistance genes: bla $_{\text {TEM-1B }}$, bla $_{\text {CTXM-15 }}$, bla $a_{\text {OXA-1 }}$, oqxB, oqxA, fosA, aac(3)lld, $a a c\left(6^{\prime}\right) l b$-cr, catB4, tet (D), dfrA14, and acrR. Although most isolates harbored the resistance genes for fosfomycin, only three were susceptible to fosfomycin (Table 1).

\section{Molecular typing and phylogenetic analysis}

All isolates were subjected to whole-genome sequencing to analyze the phylogenetic relationship and molecular characteristics. The E. coli DO7785 isolate belonged to ST69 and serotype $\mathrm{O} 153: \mathrm{H} 2$, whereas all $K$. pneumoniae isolates belonged to ST76. The urinary K. pneumoniae isolates belonged to ST36 and ST101. The K. pneumoniae isolates from patients with IE and the isolate 648 from patients with UTI belonged to ST76. All K. pneumoniae isolates were classified into the serotype $\mathrm{K} 2$. The urinary isolate 648 and the blood K. pneumoniae isolates (from patients with IE) were closely related although they infected different sites. The cgMLST scheme revealed that the clustering of the isolate 648 and the K. pneumoniae isolates from patients with IE was distinct from that of other urinary $K$. pneumoniae isolates. Similarly, E. coli DO7785 clustered with the urinary reference strain UMN26, which indicated a high degree of genetic relatedness between these two strains (Fig. 1).

\section{Plasmid and virulence gene analyses}

The presence of plasmids in E. coli and K. pneumoniae isolates was examined. In the E. coli isolate DO7785, a large

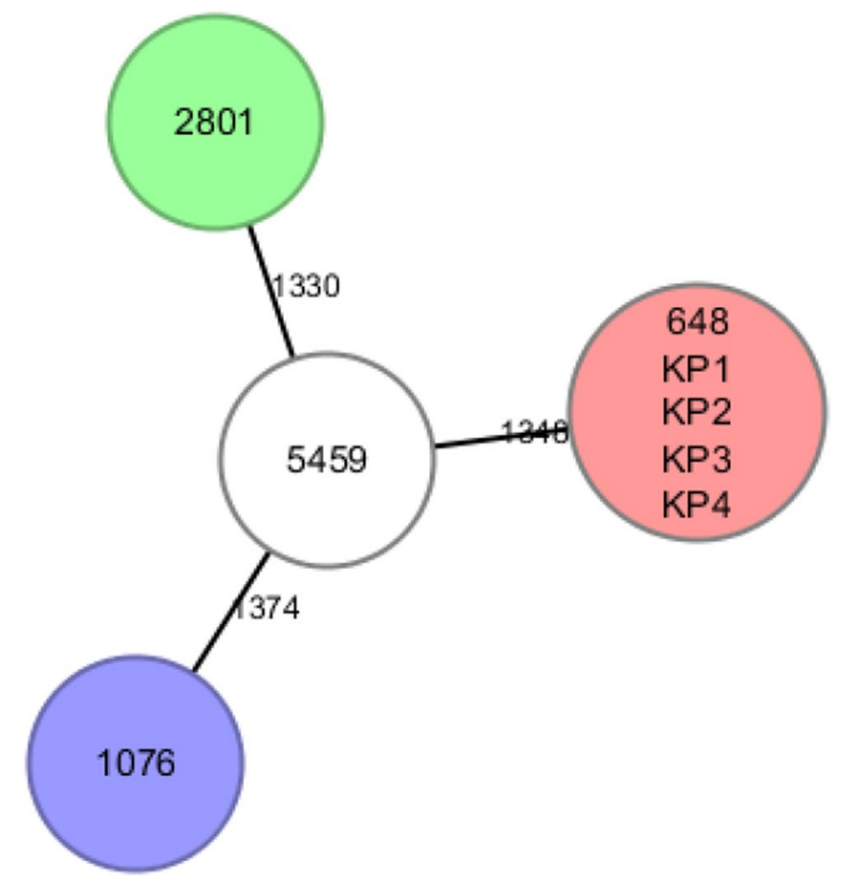

Fig. 1 Neighbor-joining (NJ) phylogenetic trees of isolates. The results using cgMLST based on 2358 genes showed the KP isolates (from endocarditis) and the 648 isolate (from urine), in red, belongs to the ST76 type. In green, the isolate 2801 (from urine) belongs to the ST101. In purple, the isolate 1076 (from urine) belongs to ST36. In white is 5459, another K. pneumoniae isolate from urine for which it was not possible to identify the ST type. The figure also showed the distance in genes between the $K$. pneumoniae isolates

plasmid sequence $(95,458 \mathrm{bp})$ containing two replicon types (IncFIB and IncFII) was identified. All K. pneumoniae isolates and the isolate 648 harbored the same reconstructed plasmid containing the replicon type Incl1, whereas the isolate 2801 harbored a different plasmid that contained both IncFIB and IncFII replicons. The other two K. pneumoniae isolates (5459 and 1076) did not harbor any plasmid. Interestingly, the plasmids in E. coli DO7785 and K. pneumoniae 2801 did not harbor any resistance genes. However, the IncFIA plasmids in the K. pneumoniae isolates and the closely related isolate 648 contained beta-lactam antibiotic resistance genes. Additionally, virulence genes were identified in the plasmids, including genes involved in copper resistance and iron uptake (see Supplementary Data S4 for complete plasmid sequences). Eight of the 17 virulence genes identified were present in the study isolates. All isolates contained mrkD, urea, uge, pgaC, fimC, ompA, and hgpA. In contrast, $y b t S$ and $\operatorname{irp} 2$ were detected only in the isolate 2801 . The urinary and blood isolates exhibited identical virulence gene profiles (see Supplementary Material S5). 


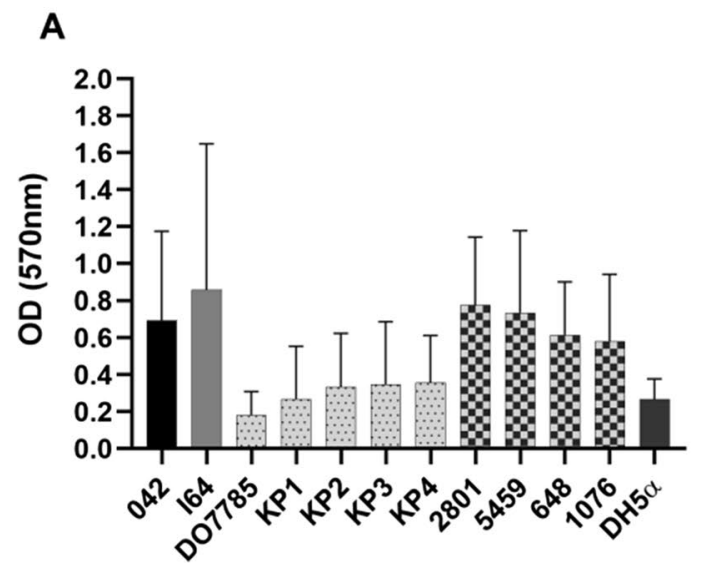

Fig. 2 Quantitative biofilm results. (A) The strains indicated in light grey with dots are isolates from the blood whereas the strains indicated in dark grey and squares are from urine samples. The strains 042 (EAEC) and I64 (UPEC) are positive controls, whereas the strain $\mathrm{DH} 5 \alpha$ is a negative control. The strains were divided into categories based upon the previously calculated OD values $\mathrm{OD} \leq \mathrm{ODc}=$ no biofilm producer; $\mathrm{ODc}<\mathrm{OD} \leq 2 \mathrm{xODc}=$ weak bio-

\section{Quantification of biofilm formation}

The biofilm-forming ability of the clinical isolates was examined. The E. coli DO7785 isolate could not form biofilms. $\mathrm{KP} 1, \mathrm{KP} 2, \mathrm{KP}$, and KP4 exhibited a weak biofilm-forming ability. In contrast, the genetically closely related isolate 2801 exhibited a moderate biofilm-forming ability (Fig. 2A). Compared with the blood isolates, the urinary isolates formed significantly stronger biofilms $(p<0.0001)$. Among the urinary isolates, the isolate 5459 exhibited a strong biofilm-forming ability, whereas the isolates 648 and 1076 exhibited a moderate biofilm-forming ability. These results indicated that the urinary isolates exhibited a higher biofilm-forming ability than the blood isolates (Fig. 2B).

\section{Adherence of Escherichia coli and Klebsiella pneumoniae strains to Vero cell and HEp-2 cell lineages}

Adherence assays were performed using the urothelial and laryngeal epithelial cells with the positive control strains EAEC042 and UPECI64. The E. coli isolate DO7785 adhered to the Vero and HEp-2 cell lines. All K. pneumoniae blood isolates (KP1, KP2, KP3, and KP4) adhered to the Vero and HEp- 2 cell lines. Additionally, the adherence ability of $K$. pneumoniae urinary isolates to these cell lines was examined. The isolates 2801 and 1076 adhered to the epithelial cells, while the isolate 5459 poorly adhered to the epithelial cells. The isolate 648 did not adhere to the Vero or HEp-2 cells (See Supplementary Data S6 for the data on pathogen adherence to Vero and HEp-2 cells) (Table 1). film producer; $2 \mathrm{xODc}<\mathrm{OD} \leq 4 \mathrm{xODc}=$ moderate biofilm producer; $4 \mathrm{xODc}<\mathrm{OD}=$ strong biofilm producer, being the ODc the OD values for the negative control strain. (B) Comparison between the isolates from blood (IE) and the isolates from urine (UTI). The asterisk indicates a statistically significant difference between all the isolates from blood and urine $(\mathrm{p}<0.05)$

\section{Discussion}

The incidence of IE caused by the members of the family Enterobacteriaceae in community and hospital settings is rare. However, a previous prospective study reported that the incidence of IE caused by gram-negative bacteria in Rio de Janeiro, Brazil, was $8.2 \%$ in the 8-year study period [3]. Another study reported that the incidence of IE caused by gram-negative bacteria in two principal teaching hospitals in Rio de Janeiro, Brazil, was higher than that reported in other studies [2]. A prospective study in Italy reported that $E$. coli was the most common etiological agent of IE caused by nonHACEK gram-negative bacteria, followed by Pseudomonas aeruginosa and K. pneumoniae [5]. Here, the characteristics of $E$. coli isolate (DO7785) from patients with CAIE and $K$. pneumoniae isolates from patients with HAIE were compared with those of urinary isolates to understand the pathophysiology of endocarditis caused by Enterobacteriaceae.

The results of this study demonstrated that the E. coli isolate DO7785 was resistant to the four tested antibiotics. However, the E. coli isolate DO7785 did not produce extended-spectrum $\beta$-lactamase (ESBL) and was not resistant to fosfomycin. The four $K$. pneumoniae clinical isolates were resistant to only three tested antibiotics and did not produce ESBL. In contrast to $E$. coli DO7785, the four $K$. pneumoniae isolates were resistant to fosfomycin. These results were consistent with those of previous studies, which reported that ESBL-producing or carbapenemase-producing Enterobacteriaceae members rarely cause IE [24]. E. coli DO7785 exhibited resistance to ampicillin and trimethoprim/sulfamethoxazole. A previous study reported a high 
prevalence of ampicillin resistance in $E$. coli [25]. Intravenous administration of fosfomycin has been suggested as a potential treatment for infections caused by drug-resistant gram-negative bacteria, including endocarditis. Fosfomycin has been considered as an alternative antimicrobial to treat infections caused by MDR gram-negative bacteria in cases where other antibiotic treatment options have failed [26]. Most isolates in this study were classified as MDR. This was not consistent with the findings of previous studies, which reported that endocarditis caused by Enterobacteriaceae can be successfully treated using first-choice antibiotics [27, 28]. In contrast, another study reported a case of endocarditis caused by carbapenemase-resistant $K$. pneumoniae, which has raised concerns about endocarditis caused by MDR bacteria [29].

Additionally, the molecular characteristics of isolates from patients with IE and urinary isolates were comparatively analyzed. The four K. pneumoniae isolates (KP1, $\mathrm{KP} 2, \mathrm{KP} 3$, and KP4) belonged to ST76, whereas E. coli DO7785 belonged to ST69, which is commonly associated with UTI cases. The K. pneumoniae urinary isolate 648 from a patient with UTI also belonged to ST76. The cgMLST scheme revealed that the isolate 648 and the four KP isolates exhibited similar clustering patterns. Moreover, ST76 $K$. pneumoniae isolates have been previously reported to be associated with infection outbreaks in hospitals [30, 31]. These ST76 isolates exhibited the same plasmid, resistance, and virulence gene profiles. This indicated that the complex UTI is a potential source of endocarditis-causing $K$. pneumoniae in the patient. The other $K$. pneumoniae isolates belonged to ST36 and ST101, which are globally associated with drug-resistant and hypervirulent $K$. pneumoniae isolates from hospitalized patients with UTIs. ST101 and ST36 infections were reported to be the etiological agents for invasive infections in humans [32]. The molecular characteristics of E. coli DO7785 were compared with those of the reference urinary isolate obtained from a public database. The results of this study revealed similarities between the blood E. coli isolate from a patient with IE and a urinary isolate belonging to the same ST. In contrast to the findings of previous studies, this study reported that the isolates do not harbor $r m p A$, which is detected in hypervirulent $K$. pneumoniae belonging to the same ST. Additionally, the isolates did not exhibit the same drug resistance profile as that of the hypervirulent $K$. pneumoniae [33]. This indicated that the isolates are not hypervirulent and that they do not produce ESBL, which can be a major threat to human health as they can cause invasive infections [34].

The $E$. coli DO7785 isolate harbored genes associated with aminoglycoside resistance (strA, strB, and aadA5), beta-lactam resistance $\left(b l a_{T E M-1 B}\right)$, sulfonamide resistance (sull and sul2), and trimethoprim resistance (dfrAl7). Araby et al. (2015) [35] demonstrated that sulfonamide resistance genes were prevalent among ESBL-producing E. coli and non-ESBL-producing $E$. coli with a high frequency of bla $_{\text {TEM }}$ [35]. $d f r A 17$, which was expressed in the isolate $E$. coli DO7785, is commonly found in Korea [36]. All clinical $K$. pneumoniae isolates exhibited similar resistance gene profiles (beta-lactamase resistance $\left(b l a_{S H V-1}\right)$, fosfomycin resistance, and $o q x A$ and $o q x B$ ). These results are consistent with the resistance phenotype. All $K$. pneumoniae urinary isolates contained the same quinolone resistance genes $(o q x A$, and $o q x B)$. The most important mechanism of quinolone resistance is aberrations in the chromosomal regions encoding quinolone resistance [37]. Some studies have suggested that $o q x A$ and $o q x B$ are conserved in the $K$. pneumoniae chromosomes [38-40]. However, Matinez et al. [41] reported that $o q x A$ and $o q x B$ are encoded in a large plasmid $(<160 \mathrm{~kb})$ [41]. The presence of $o q x A$ and $o q x B$ in the plasmids in $K$. pneumoniae can be explained by their capture from the chromosomal genome, which may be a reservoir for this antibiotic resistance $[38,39]$. The activity of ESBLs is correlated with fluoroquinolone resistance [42]. The presence of ESBL and some fluoroquinolone-resistant genes in the same mobile genetic elements may result in coresistance to $\beta$-lactams and fluoroquinolones [43]. Azargun et al. [42] demonstrated that resistance to fluoroquinolones (89.3\%) in ESBL-producing isolates was significantly higher than that in non-ESBL-producing isolates. This indicated that $o q x B$ and $o q x A$ are detected in a significant proportion of ESBL-producing Enterobacteriaceae [42].

Although the urinary K. pneumoniae isolate 1076 contained fos $A 5$ and quinolone resistance genes, it was resistant to only amoxicillin (owing to the presence of $b l a_{S H V I 1}$, a narrow-spectrum beta-lactamase). In this study, two urinary K. pneumoniae isolates (2801 and 648) harbored ESBLencoding genes belonging to the CTX-M group. The genes located on the plasmids can be easily transmitted [44]. Quinolone resistance among $K$. pneumoniae clinical isolates is a serious health concern as ciprofloxacin is widely prescribed as a broad-spectrum antimicrobial agent for the treatment of UTI caused by ESBL-producing $K$. pneumoniae [45]. Some plasmids co-exist with $b l a_{C T X M}$ and $a a c\left(6^{\prime}\right)-I b-c r$, which promote resistance to cephalosporins and fluoroquinolones [35].

The presence of virulence genes was also investigated in this study because of their importance in the pathogenesis of E. coli and K. pneumoniae infections. The urease virulence factor, which is critical for catheter encrustation, is encoded in all $K$. pneumoniae from patients with IE and UTIs. Other virulence genes such as uge, fimC, and hgpA identified in these isolates are reported to be associated with the pathogenesis of UTIs [46-48]. Several Enterobacteriaceae species form biofilms, which further contribute to the resistance to antimicrobial agents. Biofilms are also associated with device-related infections, including endocarditis and UTIs [49]. In this study, all urinary isolates could form biofilms. 
In contrast, the blood E. coli DO7785 isolate could not form biofilms. Meanwhile, the KP1, KP2, KP3, and KP4 isolates formed weak biofilms. Interestingly, the urinary isolate 2801 , which is closely related to the clinical $K$. pneumoniae isolates, formed significantly stronger biofilms than the blood $K$. pneumoniae isolates.

The results of the molecular assay revealed that all strains expressed important virulence genes associated with biofilm formation. $m r k D$, an operon that encodes type 3 fimbriae, plays an important role in biofilm formation [50-53]. The presence of $f i m C$, which encodes the chaperone FimC, was also detected. FimC is part of a complex group of adhesion molecules from type 1 fimbriae that is involved in adherence to the host cells and biofilm formation [46]. E. coli DO7785 expresses a virulence gene that is commonly present in uropathogenic Escherichia coli (UPEC). fimH encodes the protein FimH, which is localized at chaperone fimbriae type 1 and promotes the bacterial adherence to the host bladder. Additionally, FimH is involved in biofilm formation and adherence to urinary tissue $[54,55]$.

Previous epidemiological studies have reported that the low frequency of IE caused by Enterobacteriaceae can be attributed to of inability of gram-negative bacteria to attach to the cardiac valves. In contrast, typical gram-positive pathogens and gram-negative HACEK bacterial group can bind to the cardiac valves [5]. In this study, all strains associated with IE adhered to the HEp-2 and Vero cell lines. Among the isolates associated with urinary infections, three $K$. pneumoniae strains exhibited strong adherence, one K. pneumoniae strain exhibited poor adherence, and one K. pneumoniae could not adhere to the HEp-2 and Vero cell lines. These results suggest that the adherence ability of Enterobacteriaceae isolates from patients with IE was different from that reported in previous studies [5]. E. coli (DO7785) and all K. pneumoniae strains express OmpA, which is an outer membrane porin that is associated with adherence to Vero and HEp-2 cell lines. In addition to encoding the major porin on the outer membrane of gram-negative bacteria, OmpA is involved in the pathogenic mechanisms, such as inhibition of proinflammatory cytokine production in isolated monocytes, induction of cell death, and adherence, invasion, and persistence to host cells [56-58].

\section{Conclusions}

The results of this study revealed that the molecular characteristics of E. coli and K. pneumoniae, which cause endocarditis, are similar to those of isolates from UTI cases. The virulence and drug resistance profiles of these isolates indicate that they can infect both the urinary system and the cardiovascular system. These isolates belong to STs that are considered a threat to human health. Although the isolates did not exhibit the same resistance and virulence gene profiles as those reported in hypervirulent clones, they can cause invasive infections. The genetically similar isolates did not exhibit the same virulence phenotype. Phenotypic analysis was performed to analyze the pathogenicity of the strains. The isolates formed biofilms and adhered to the host cells, which indicated that they are pathogenic. These results suggest that urine is the source of isolates from patients with IE and that these isolates are not similar to hypervirulent clones. Hence, these isolates must be monitored as they can cause complex infections in susceptible hosts. The limitations of this study include the small sample size. Further studies are necessary to clarify the molecular profile of $E$. coli and K. pneumoniae strains from patients with UTIs that can cause IE.

Supplementary Information The online version contains supplementary material available at https://doi.org/10.1007/s42770-021-00528-w.

Funding This study was partly funded by the Coordenação de Aperfeiçoamento de Pessoal de Nível Superior-Brasil (CAPES)—Finance Code 001. Nathália L. Andrade was supported by Fundação de Amparo a Pesquisa do Estado do Rio de Janeiro - FAPERJ (Process number: E-26/202.743/2017).

\section{Declarations}

Ethical statement This study was reviewed and approved by the Institutional Board of the Committee of Pedro Ernesto University Hospital (CAAE: 01247512.3.0000.5259).

Conflict of interest The authors declare no competing interests.

Open Access This article is licensed under a Creative Commons Attribution 4.0 International License, which permits use, sharing, adaptation, distribution and reproduction in any medium or format, as long as you give appropriate credit to the original author(s) and the source, provide a link to the Creative Commons licence, and indicate if changes were made. The images or other third party material in this article are included in the article's Creative Commons licence, unless indicated otherwise in a credit line to the material. If material is not included in the article's Creative Commons licence and your intended use is not permitted by statutory regulation or exceeds the permitted use, you will need to obtain permission directly from the copyright holder. To view a copy of this licence, visit http://creativecommons.org/licenses/by/4.0/.

\section{References}

1. Habib G, Lancellotti P, Antunes MJ, Bongiorni MG, Casalta JP et al (2015) 2015 ESC guidelines for the management of infective endocarditis: the task force for the management of infective endocarditis of the European Society of Cardiology (ESC). Eur Heart J 36:3075-3128. https://doi.org/10.1093/eurheartj/ehv319

2. Damasco PV, Ramos JN, Correal JCD, Potsch MV, Vieira VV et al (2014) Infective endocarditis in Rio de Janeiro, Brazil: a 5-year experience at two teaching hospitals. Infection 42:835-842. https://doi.org/10.1007/s15010-014-0640-2 
3. Damasco PV, Correal JCD, Cruz-Campos ACD, Wajsbrot BR, Cunha RGD et al (2019) Epidemiological and clinical profile of infective endocarditis at a Brazilian tertiary care center: an eightyear prospective study. Rev Soc Bras Med Trop 52:e2018375. https://doi.org/10.1590/0037-8682-0375-2018

4. Raza SS, Sultan OW, Sohail MR (2010) Gram-negative bacterial endocarditis in adults: state-of-the-heart. Expert Rev Anti Infect Ther 8:879-885. https://doi.org/10.1586/eri.10.76

5. Falcone M, Tiseo G, Durante-Mangoni E, Ravasio V, Barbaro F (2018) Risk factors and outcomes of endocarditis due to nonHACEK gram-negative bacilli: data from the prospective multicenter Italian endocarditis study cohort. Antimicrob Agents Chemother 62:e2208-e2217. https://doi.org/10.1128/AAC. 02208-17

6. Compain F, Babosan A, Brisse S, Genel N, Audo J et al (2014) Multiplex PCR for detection of seven virulence factors and K1/ K2 capsular serotypes of Klebsiella pneumoniae. J Clin Microbiol 52:4377-4380. https://doi.org/10.1128/JCM.02316-14

7. Campos ACC, Andrade NL, Ferdous M, Chlebowicz MA, Santos CC et al (2018) Comprehensive molecular characterization of Escherichia coli isolates from urine samples of hospitalized patients in Rio de Janeiro. Brazil Front Microbiol 9:243. https://doi.org/10.3389/ fmicb.2018.00243

8. Saltoglu N, Karali R, Yemisen M, Ozaras R, Balkan II et al (2015) Comparison of community-onset healthcare-associated and hospital-acquired urinary infections caused by extended-spectrum betalactamase-producing Escherichia coli and antimicrobial activities. Int J Clin Pract 69:766-770. https://doi.org/10.1111/ijcp.12608

9. Loubet P, Lescure FX, Lepage L, Kirsch M, Armand-Lefevre L et al (2015) Endocarditis due to gram-negative bacilli at a French teaching hospital over a 6-year period: Clinical characteristics and outcome. Infect Dis (Lond) 47:889-895. https:// doi.org/10.3109/23744235.2015.1075660

10. Martinez P, Garzón D, Mattar S (2012) CTX-M-producing Escherichia coli and Klebsiella pneumoniae isolated from community-acquired urinary tract infections in Valledupar. Colombia Braz J Infect Dis 16:420-425. https://doi.org/10.1016/j.bjid. 2012.05.001

11. Ríos E, López MC, Rodríguez-Avial I, Culebras E, Picazo JJ (2017) Detection of Escherichia coli ST131 clonal complex (ST705) and Klebsiella pneumoniae ST15 among faecal carriage of extended-spectrum $\beta$-lactamase- and carbapenemaseproducing Enterobacteriaceae. J Med Microbiol 66:169-174. https://doi.org/10.1099/jmm.0.000399

12. Sheikh J, Hicks S, Dall'Agnol M, Phillips AD, Nataro JP (2001) Roles for Fis and YafK in biofilm formation by enteroaggregative Escherichia coli. Mol Microbiol 41:983-997. https://doi. org/10.1046/j.1365-2958.2001.02512.x

13. Magiorakos AP, Srinivasan A, Carey RB, Carmeli Y, Falagas ME et al (2012) Multidrug-resistant, extensively drug-resistant and pandrug-resistant bacteria: An international expert proposal for interim standard definitions for acquired resistance. Clin Microbiol Infect 18:268-281. https://doi.org/10.1111/j.14690691.2011.03570.x

14. Aziz RK, Bartels D, Best AA, DeJongh M, Disz T et al (2008) The RAST server: rapid annotations using subsystems technology. BMC Genomics 9:75. https://doi.org/10.1186/ 1471-2164-9-75

15. Larsen MV, Cosentino $\mathrm{S}$, Rasmussen $\mathrm{S}$, Friis $\mathrm{C}$, Hasman $\mathrm{H}$ et al (2012) Multilocus sequence typing of total-genome-sequenced bacteria. J Clin Microbiol 50:1355-1361. https://doi.org/10.1128/ JCM.06094-11

16. Zankari E, Hasman H, Cosentino S, Vestergaard M, Rasmussen $S$ et al (2012) Identification of acquired antimicrobial resistance genes. J Antimicrob Chemother 67:2640-2644. https://doi.org/10. 1093/jac/dks261
17. Wick RR, Heinz E, Holt KE, Wyres KL (2018) Kaptive web: User-friendly capsule and lipopolysaccharide serotype prediction for Klebsiella genomes. J Clin Microbiol 56:e00197-e218. https:// doi.org/10.1128/JCM.00197-18

18. Joensen KG, Tetzschner AMM, Iguchi A, Aarestrup FM, Scheutz F (2015) Rapid and easy in silico serotyping of Escherichia coli isolates by use of whole-genome sequencing data. J Clin Microbiol 53:2410-2426. https://doi.org/10.1128/JCM.00008-15

19. Carattoli A, Zankari E, Garcia-Fernandez A, Larsen MV, Lund O et al (2014) In silico detection and typing of plasmids using PlasmidFinder and plasmid multilocus sequence typing. Antimicrob Agents Chemother 58:3895-3903. https://doi.org/10.1128/AAC. 02412-14

20. Robertson J, Nash JHE (2018) MOB-suite: Software tools for clustering, reconstruction and typing of plasmids from draft assemblies. Microb Genom 4:e000206. https://doi.org/10.1099/mgen.0. 000206

21. Nataro JP, Deng Y, Cookson S, Cravioto A, Savarino SJ et al (1995) Heterogeneity of enteroaggregative Escherichia coli virulence demonstrated in volunteers. J Infect Dis 171:465-468. https://doi.org/10.1093/infdis/171.2.465

22. Rosa AC, Mariano AT, Pereira AM, Tibana A, Gomes TA et al (1998) Enteropathogenicity markers in Escherichia coli isolated from infants with acute diarrhoea and healthy controls in Rio de Janeiro. Brazil J Med Microbiol 47:781-790. https://doi.org/10. 1099/00222615-47-9-781

23 Stepanović S, Vuković D, Hola V, Di Bonaventura G, Djukić S et al (2007) Quantification of biofilm in microtiter plates: overview of testing conditions and practical recommendations for assesment of biofilm production by staphylococci. APMIS 115:8919. https://doi.org/10.1111/j.1600-0463.2007.apm_630.x

24. Kim CJ, Yi JE, Kim Y, Choi HJ (2018) Emphysematous endocarditis caused by AmpC beta-lactamase-producing Escherichia coli: a case report. Medicine (Baltimore) 97:e9620. https://doi.org/10. 1097/MD.0000000000009620

25. Esteve-Palau E, Solande G, Sánchez F, Sorlí L, Montero M et al (2015) Clinical and economic impact of urinary tract infections caused by ESBL-producing Escherichia coli requiring hospitalization: a matched cohort study. J Infect 71:667-674. https://doi.org/ 10.1016/j.jinf.2015.08.012

26. Zhanel GG, Walkty AJ, Karlowsky JA (2016) Fosfomycin: A firstline oral therapy for acute uncomplicated cystitis. Can J Infect Dis Med Microbiol 2016:2082693. https://doi.org/10.1155/2016/ 2082693

27. Aubron C, Charpentier J, Trouillet JL, Offenstadt G, Mercat A et al (2006) Native-valve infective endocarditis caused by Enterobacteriaceae: report on 9 cases and literature review. Scand J Infect Dis 38:873-881. https://doi.org/10.1080/003655406007404 88

28 Reyes MP, Reyes KC (2008) Gram-negative endocarditis. Curr Infect Dis Rep 10:267-74. https://doi.org/10.1007/ s11908-008-0044-5

29. Benenson S, Navon-Venezia S, Carmeli Y, Adler A, Strahilevitz J et al (2009) Carbapenem-resistant Klebsiella pneumoniae endocarditis in a young adult: successful treatment with gentamicin and colistin. Int J Infect Dis 13:e295-e298. https://doi.org/10. 1016/j.ijid.2009.01.006

30. Zhu J, Sun L, Ding B, Yang Y, Xu X et al (2016) Outbreak of NDM-1-producing Klebsiella pneumoniae ST76 and ST37 isolates in neonates. Eur J Clin Microbiol Infect Dis 35:611-618. https://doi.org/10.1007/s10096-016-2578-Z

31. Zhang X, Chen D, Xu G, Huang W, Wang X (2018) Molecular epidemiology and drug resistant mechanism in carbapenemresistant Klebsiella pneumoniae isolated from pediatric patients in Shanghai. China PLoS One 13:e0194000. https://doi.org/10. 1371/journal.pone.0194000 
32. Shankar C, Shankar BA, Manesh A, Veeraraghavan B (2018) KPC-2 producing ST101 Klebsiella pneumoniae from bloodstream infection in India. J Med Microbiol 67:927-930. https:// doi.org/10.1099/jmm.0.000767

33. Feng Y, Lu Y, Yao Z, Zong Z (2018) Carbapenem-resistant hypervirulent Klebsiella pneumoniae of sequence type 36. Antimicrob Agents Chemother 62:e02644-e2717. https://doi.org/10.1128/ AAC.02644-17

34. Holt KE, Wertheim H, Zadoks RN, Baker S, Whitehouse CA et al (2015) Genomic analysis of diversity, population structure, virulence, and antimicrobial resistance in Klebsiella pneumoniae, an urgent threat to public health. Proc Natl Acad Sci U S A 112:E3574-E3581. https://doi.org/10.1073/pnas.1501049112

35. Arabi H, Pakzad I, Nasrollahi A, Hosainzadegan H, Jalilian FA et al (2015) Sulfonamide resistance genes (sul) $M$ in extendedspectrum $\beta$-lactamase (ESBL) and non-ESBL producing Escherichia coli isolated from Iranian hospitals. Jundishapur J Microbiol 8:e19961. https://doi.org/10.5812/jjm.19961v2

36. Yu HS, Lee JC, Kang HY, Jeong YS, Lee EY et al (2004) Prevalence of $d f r$ genes associated with integrons and dissemination of $d f r A 17$ among urinary isolates of Escherichia coli in Korea. J Antimicrob Chemother 53:445-450. https://doi.org/10.1093/jac/ dkh097

37. Ni Q, Tian Y, Zhang L, Jiang C, Dong D et al (2016) Prevalence and quinolone resistance of fecal carriage of extended-spectrum $\beta$-lactamase-producing Escherichia coli in 6 communities and 2 physical examination Center populations in Shangai. China Diagn Microbiol Infect Dis 86:428-433. https://doi.org/10.1016/j.diagm icrobio.2016.07.010

38. Norman A, Hansen LH, She Q, Sorensen SJ (2008) Nucleotide sequence of pOLA52: a conjugative IncX1 plasmid from Escherichia coli which enables biofilm formation and multidrug efflux. Plasmid 60:59-74. https://doi.org/10.1016/j.plasmid.2008.03.003

39. Kim HB, Wang M, Park CH, Kim EC, Jacoby GA et al (2009) oqx $\mathrm{AB}$ encoding a multidrug efflux pump in human clinical isolates of Enterobacteriaceae. Antimicrob Agents Chemother 53:3582-3584. https://doi.org/10.1128/AAC.01574-08

40. Yuan J, Xu X, Guo Q, Zhao X, Ye X et al (2012) Prevalence of the oqxAB gene complex in Klebsiella pneumoniae and Escherichia coli clinical isolates. J Antimicrob Chemother 67:1655-1659. https://doi.org/10.1093/jac/dks086

41. Rodríguez-Martínez JM, Díaz de Alba P, Briales A, Machuca J, Lossa M et al (2013) Contribution of OqxAB efflux pumps to quinolone resistance in extended-spectrum-b-lactamase-producing Klebsiella pneumoniae. J Antimicrob Chemother 68:68-73. https://doi.org/10.1093/jac/dks377

42. Azargun R, Sadeghi MR, Barhaghi MHS, Kafil HS, Yeganeh F et al (2018) The prevalence of plasmid-mediated quinolone resistance and ESBL-production in Enterobacteriaceae isolated from urinary tract infections. Infect Drug Resist 11:1007-1014. https:// doi.org/10.2147/IDR.S160720

43. Yadav KK, Adhikari N, Khadka R, Pant AD, Shah B (2015) Multidrug resistant Enterobacteriaceae and extended spectrum betalactamase producing Escherichia coli: a cross-sectional study in National Kidney Center. Nepal Antimicrob Resist Infect Control 4:42. https://doi.org/10.1186/s13756-015-0085-0

44. Sharma J, Sharma M, Ray P (2010) Detection of TEM \& SHV genes in Escherichia coli \& Klebsiella pneumoniae isolates in a tertiary care hospital from India. Indian J Med Res 132:332-336

45. Goudarzi M, Azad M, Seyedjavadi SS (2015) Prevalence of plasmid-mediated quinolone resistance determinants and $\mathrm{OqxAB}$ efflux pumps among extended-spectrum $\beta$-lactamase producing
Klebsiella pneumoniae isolated from patients with nosocomial urinary tract infection in Tehran. Iran Scientifica (Cairo) 2015:518167. https://doi.org/10.1155/2015/518167

46. Jones CH, Pinkner JS, Roth R, Heuser J, Nicholes AV et al (1995) FimH adhesin of type 1 pili is assembled into a fibrillar tip structure in the Enterobacteriaceae. Proc Natl Acad Sci U S A 92:2081-2085. https://doi.org/10.1073/pnas.92.6.2081

47. Lin WH, Tseng CC, Wu AB, Yang DC, Cheng SW et al (2015) Clinical and microbiological characteristics of peritoneal dialysisrelated peritonitis caused by Klebsiella pneumoniae in southern Taiwan. J Microbiol Immunol Infect 48:276-283. https://doi.org/ 10.1016/j.jmii.2013.10.002

48. Di Martino P, Cafferini N, Joly B, Darfeuille-Michaud A (2003) Klebsiella pneumoniae type 3 pili facilitate adherence and biofilm formation on abiotic surfaces. Res Microbiol 154:9-16. https:// doi.org/10.1016/s0923-2508(02)00004-9

49. de la Fuente-Núñez C, Reffuveille F, Fernández L, Hancock REW (2013) Bacterial biofilm development as a multicellular adaptation: antibiotic resistance and new therapeutic strategies. Curr Opin Microbiol 16:580-589. https://doi.org/10.1016/j.mib.2013. 06.013

50. Paterson DL, Mulazimoglu L, Casellas JM, Ko WC, Goossens H et al (2000) Epidemiology of ciprofloxacin resistance and its relationship to extended spectrum-lactamase production in Klebsiella pneumoniae isolates causing bacteremia. Clin Infect Dis 30:473-478. https://doi. org/10.1086/313719

51. Jagnow J, Clegg S (2003) Klebsiella pneumoniae MrkD-mediated biofilm formation on extracellular matrix- and collagen-coated surfaces. Microbiology (Reading) 149:2397-2405. https://doi.org/ 10.1099/mic.0.26434-0

52. Clegg S, Murphy CN (2016) Epidemiology and virulence of Klebsiella pneumoniae. Microbiol Specr 4:1-17. https://doi.org/10. 1128/microbiolspec.UTI-0005-2012

53. Mao BH, Chang YF, Scaria J, Chang CC, Chou LW et al (2012) Identification of Escherichia coli genes associated with urinary tract infections. J Clin Microbiol 50:449-456. https://doi.org/10. 1128/JCM.00640-11

54. Norinder BS, Koves B, Yadav M, Brauner A, Svanborg C (2012) Do Escherichia coli strains causing acute cystitis have a distinct virulence repertoire? Microb Pathog 52:10-16. https://doi.org/10. 1016/j.micpath.2011.08.005

55. Wu XR, Sun TT, Medina JJ (1996) In vitro binding of the typi 1-fimbriated Escherichia coli to uroplakins Ia and Ib: Relation to urinary tract infections. Proc Natl Acad Sci U S A 93:9630-9635. https://doi.org/10.1073/pnas.93.18.9630

56. Kwon HII, Kim S, Oh MH, Na SH, Kim YJ et al (2017) Outer membrane protein A contributes to antimicrobial resistance of Acinetobacter baumannii through the OmpA-like domain. J Antimicrob Chemother 72:3012-3015. https://doi.org/10.1093/jac/ $\mathrm{dkx} 257$

57. Selvaraj SK, Prasadarao NV (2005) Escherichia coli K1 inhibits proinflammatory cytokine induction in monocytes by preventing NF- $\kappa B$ activation. J Leukoc Biol 78:544-554. https://doi.org/10. 1189/jlb.0904516

58. Sato Y, Unno Y, Kawkami S, Ubagai T, Ono Y (2017) Virulence characteristics of Acinetobacter baumannii clinical isolates vary with the expression levels of omps. J Med Microbiol 66:203-212. https://doi.org/10.1099/jmm.0.000394

Publisher's note Springer Nature remains neutral with regard to jurisdictional claims in published maps and institutional affiliations. 